\title{
Follow-up of potential novel Graves' disease susceptibility loci, identified in the UK WTCCC genome-wide nonsynonymous SNP study
}

\author{
Paul R Newby ${ }^{1}$, Oliver J Pickles ${ }^{1}$, Samaresh Mazumdar ${ }^{1}$, Oliver J Brand ${ }^{1}$, Jaqueline D Carr-Smith ${ }^{1}$, \\ Simon HS Pearce ${ }^{2}$, Jayne A Franklyn ${ }^{1}$, Wellcome Trust Case-Control Consortium (WTCCC) ${ }^{3}$, \\ David M Evans ${ }^{4}$, Matthew J Simmonds ${ }^{1,5}$ and Stephen CL Gough ${ }^{\star, 1,5}$
}

\begin{abstract}
A recent association scan using a genome-wide set of nonsynonymous coding single-nucleotide polymorphisms (nsSNPs) conducted in four diseases including Graves' disease (GD), identified nine novel possible regions of association with GD. We used a case-control approach in an attempt to replicate association of these nine regions in an independent collection of 1578 British GD patients and 1946 matched Caucasian controls. Although none of these loci showed evidence of association with GD in the independent data set, when combined with the original Wellcome Trust Case-Control Consortium study group, minor differences in allele frequencies $\left(P \geqslant 10^{-3}\right)$ remained in the combined collection of 5924 subjects for four of the nsSNPs, present within HDLBP, TEKT1, JSRP1 and UTX. An additional 29 Tag SNPs were screened within these four gene regions to determine if further associations could be detected. Similarly, minor differences only $(P=0.042-0.002)$ were detected in two HDLBP and two TEKT1 Tag SNPs in the combined UK GD collection. In conclusion, it is unlikely that the SNPs selected in this replication study have a significant effect on the risk of GD in the United Kingdom. Our study confirms the need for large data sets and stringent analysis criteria when searching for susceptibility loci in common diseases.
\end{abstract} European Journal of Human Genetics (2010) 18, 1021-1026; doi:10.1038/ejhg.2010.55; published online 5 May 2010

Keywords: Graves' disease; nonsynonymous SNPs; genome-wide screening

\section{INTRODUCTION}

Autoimmune thyroid diseases (AITD) including Graves' disease (GD) and Hashimoto's thyroiditis are common autoimmune diseases that develop as a result of environmental triggers in individuals with a genetic predisposition. Although a number of replicated genetic associations are emerging, providing insights into the underlying disease mechanisms, a significant component of the genetic contribution to AITD remains unknown.

In keeping with other common diseases, the identification of novel genetic variants conferring susceptibility has proved problematic with genome-wide linkage analysis generally proving disappointing in identifying loci for AITD. ${ }^{1,2}$ Recent genome-wide association studies are, however, now beginning to reveal a number of novel genetic variants in many common diseases. The Wellcome Trust Case-Control Consortium (WTCCC) in the United Kingdom has recently completed two large association studies in 11 common diseases and reported a number of novel loci for most diseases. ${ }^{3,4}$ In the smaller of the two studies, the WTCCC investigated 5500 individuals, which included 900 cases with GD, using a genome-wide set of 14500 nonsynonymous coding single-nucleotide polymorphisms (nsSNPs). Although the strongest association signal was unsurprisingly identified in the human leukocyte antigen (HLA) region $\left(P\right.$ value $\left.<10^{-20}\right)$, association was also confirmed at the previously reported thyroid stimulating hormone receptor gene $(T S H R)^{5}$ and Fc receptor-like 3 gene (FCRL3), ${ }^{4,6}$ with a further nine novel regions showing some evidence of association with $P$ value $\leqslant 10^{-4}$. The aim of this study was to try to replicate the association identified in the nine novel regions in an independent UK collection of GD subjects and controls.

\section{MATERIALS AND METHODS}

Subjects

A total cohort of 2478 unrelated Caucasian GD patients of UK origin were recruited into the AITD UK National Collection as previously described. ${ }^{7}$ Control samples totaling 3446 were obtained from the 1958 British Birth cohort (http://www.b58cgene.sgul.ac.uk). The WTCCC had previously genotyped $900 \mathrm{GD}$ patients and 1500 control subjects from these data sets in the 14500 nsSNPs association scan. ${ }^{4}$ In total, therefore, a further 1578 GD patients and 1946 controls, not previously included in the WTCCC nsSNP scan, were incorporated into this replication study. All subjects gave informed written consent and the project was approved by the local research ethics committee.

\section{nsSNP genotyping}

Nine novel nsSNP associations detected outside the HLA region that met a point-wise significance level of $P \leqslant 10^{-4}$ in the original WTCCC nsSNP scan were genotyped in an independent collection of 3524 samples (see Table 1 for a

\footnotetext{
${ }^{1}$ Centre for Endocrinology, Diabetes and Metabolism, School of Clinical and Experimental Medicine, College of Medical and Dental Sciences, Institute of Biomedical Research, University of Birmingham, Birmingham, West Midlands, UK; ${ }^{2}$ Institute of Human Genetics, University of Newcastle, International Centre for Life, Newcastle upon Tyne, UK; ${ }^{3}$ See Appendix 1 for membership of the WTCCC; ${ }^{4}$ Department of Social Medicine, MRC Centre for Causal Analyses in Translational Epidemiology, University of Bristol, Bristol, UK *Correspondence: Professor SCL Gough, Oxford Centre for Diabetes, Endocrinology and Metabolism, University of Oxford, Headington, Oxford, OX3 7LJ, UK.

Tel: +44 1865 857560; Fax: +44 1865 857561; E-mail: stephen.gough@OCDEM.ox.ac.uk

5 These authors contributed equally to this work.

Received 26 October 2009; revised 3 March 2010; accepted 12 March 2010; published online 5 May 2010
} 


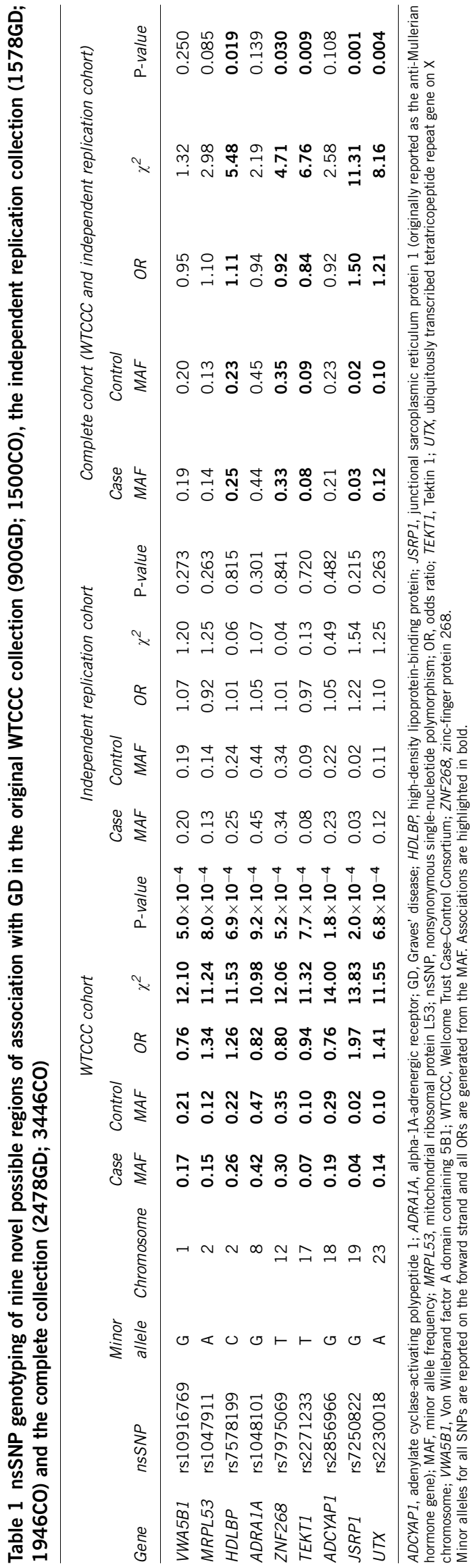

list of all SNPs investigated). The FCRL and TSHR regions were not examined as association with AITD had been previously reported. ${ }^{4-6}$ A further tenth SNP, rs3748140, present within the protein phosphatase 1 regulatory inhibitor Subunit 3B gene (PPPIR38) also produced a point-wise significance level of $P \leqslant 10^{-4}$ but was excluded from further analysis as it was non-polymorphic in the replication study.

\section{Tag SNP genotyping}

To provide greater coverage in selected candidate genes in which nsSNPs showed some evidence for association in the combined WTCCC and replication data sets we also typed a number of Tag SNPs. A total of 29 Tag SNPs were selected (excluding the initially typed nsSNPs within each gene region) to take into account all remaining known common variation within HDLBP, TEKT1, JSRP1 and UTX (see Table 2 for details of Tag selection). These Tag SNPs were genotyped in the complete GD collection of 2478 samples. The control group, however, was reduced to 2690 to ensure geographical matching of cases and controls (756 controls originally used as part of the WTCCC were not screened) and referred to as the geographically matched complete group. Assays for all of the above SNPs were purchased from Applied Biosystems, Warrington, UK and genotyped on an ABI7900HT using Taqman (Applied Biosystems) genotyping technologies.

\section{Statistical analysis}

All SNPs in HDLBP, TEKT and JSRP1 were in Hardy-Weinberg equilibrium (HWE) in cases and controls except rs10185319 (HDLBP) (control HWE $P=0.02$ ). As UTX was present on the X chromosome HWE was assessed in Haploview Version 3.2 (http://www.broad.mit.edu/mpg/haploview) and the rs6611065 SNP was shown to strongly deviate from $\operatorname{HWE}\left(P=8.64 \times 10^{-15}\right)$ and was excluded from further analysis. Allelic and genotypic analysis of case-control data was performed using the $\chi^{2}$-test within the MINITAB statistical package (MINITAB Release 15.1.2, (C) 1972-2007, Minitab Inc., State College, PA, USA). As UTX is located on the X chromosome these SNPs were analyzed within Haploview Version 3.2, which can analyze hemizygote males. Odds ratios (OR) with $95 \%$ confidence intervals (CI) were calculated by the method of Woolf with Haldane's modification for small numbers where appropriate. ${ }^{8}$ Using the ORs and minor allele frequencies (MAF) generated from the WTCCC nsSNP study, power calculations have shown that the replication collection (1578 GD and 1946 controls) had between 93 and $100 \%$ power to detect the size of effect reported from the original data set (900 GD and 1500 controls) for all loci, except TEKT1.

\section{RESULTS}

nsSNP genotyping results in the independent and complete data sets

None of the nine novel candidate nsSNPs detected within the original WTCCC study were found to be associated with GD in the independent replication collection of 1578 GD cases and 1946 controls $(P=0.215-0.841)$ (Table 1). In the combined collection (consisting of the WTCCC and the independent replication samples, totaling 2478 GD cases and 3446 controls) no significant differences in allele or genotype frequencies of the rs10916769 (VWA5B1), rs1047911 (MRPL53), rs1048101 (ADRA1A) and rs2856966 (ADCYAP1) SNPs were observed between GD cases and controls (see Table 1). However, minor differences in genotype frequencies between GD and controls persisted for, rs7578199 (HDLBP), rs2271233 (TEKT1), rs7250822 (JSRP1) and rs2230018 (UTX) SNPs, producing ORs of $1.11(95 \%$ $\mathrm{CI}=1.02-1.22), 0.84(95 \% \mathrm{CI}=0.73-0.96), 1.50 \quad(95 \% \mathrm{CI}=1.18-1.90)$ and $1.21(95 \% \mathrm{CI}=1.06-1.38)$, respectively. Differences in allele frequencies were also observed between GD cases and controls for rs7975069 (ZNF268) although no association between genotypes and GD was observed $(P=0.060)$.

On the basis of the finding of weak association of, HDLBP, TEKT1, JSRP1 and UTX in the combined collection, we then subjected the remainder of these gene regions to Tag SNP screening to capture the majority of the common variation and determine if further GD associations existed within these regions. 
Table 2 Tag SNP genotyping of HDLBP, TEKT1, JSRP1 and UTX in a case-control collection of 2478 patients with GD and 2690 control subjects

\begin{tabular}{lcccccc}
\hline Gene & Tag SNPs & Case MAF & Control MAF & P-value & OR & $95 \% \mathrm{Cl}$ \\
\hline HDLBP & rs6437249 & $\mathbf{0 . 3 0}$ & $\mathbf{0 . 3 2}$ & $\mathbf{0 . 0 3 6}$ & $\mathbf{0 . 9 1}$ & $\mathbf{0 . 8 4 - 0 . 9 9}$ \\
HDLBP & rs11680329 & $\mathbf{0 . 2 1}$ & $\mathbf{0 . 1 9}$ & $\mathbf{0 . 0 4 2}$ & $\mathbf{1 . 1 1}$ & $\mathbf{1 . 0 0 - 1 . 2 2}$ \\
HDLBP & rs3771346 & 0.25 & 0.27 & 0.082 & 0.92 & $0.84-1.01$ \\
HDLBP & rs6724257 & 0.38 & 0.39 & 0.292 & 0.96 & $0.88-1.04$ \\
HDLBP & rs2305076 & 0.09 & 0.10 & 0.364 & 0.94 & $0.82-1.07$ \\
HDLBP & rs6705421 & 0.46 & 0.45 & 0.411 & 1.03 & $0.95-1.12$ \\
HDLBP & rs15129 & 0.18 & 0.19 & 0.442 & 0.96 & $0.87-1.06$ \\
HDLBP & rs7559564 & 0.20 & 0.20 & 0.515 & 0.97 & $0.87-1.07$ \\
HDLBP & rs4675973 & 0.28 & 0.29 & 0.527 & 0.97 & $0.89-1.06$ \\
HDLBP & rs4675971 & 0.17 & 0.17 & 0.766 & 0.98 & $0.88-1.10$ \\
HDLBP & rs2289795 & 0.28 & 0.28 & 0.775 & 1.01 & $0.93-1.11$ \\
HDLBP & rs3755325 & 0.48 & 0.48 & 0.794 & 0.99 & $0.91-1.07$ \\
HDLBP & rs6757876 & 0.17 & 0.17 & 0.828 & 1.01 & $0.91-1.13$ \\
HDLBP & rs10185319 & Failed HWE & Failed HWE & - & - & -
\end{tabular}

\begin{tabular}{lcccccc} 
TEKT1 & rs4796561 & $\mathbf{0 . 4 2}$ & $\mathbf{0 . 4 5}$ & $\mathbf{0 . 0 0 2}$ & $\mathbf{0 . 8 8}$ & $\mathbf{0 . 8 1}-\mathbf{0 . 9 6}$ \\
TEKT1 & rs4796356 & $\mathbf{0 . 2 9}$ & $\mathbf{0 . 2 7}$ & $\mathbf{0 . 0 1 0}$ & $\mathbf{1 . 1 2}$ & $\mathbf{1 . 0 3}-\mathbf{1 . 2 3}$ \\
TEKT1 & rs8078571 & $\mathbf{0 . 4 6}$ & $\mathbf{0 . 4 8}$ & $\mathbf{0 . 0 3 7}$ & $\mathbf{0 . 9 1}$ & $\mathbf{0 . 8 5}-\mathbf{0 . 9 9}$ \\
TEKT1 & rs3744395 & 0.20 & 0.21 & 0.172 & 0.93 & $0.85-1.03$ \\
TEKT1 & rs17804647 & 0.10 & 0.10 & 0.241 & 1.08 & $0.95-1.24$ \\
TEKT1 & rs17731932 & 0.14 & 0.14 & 0.548 & 0.97 & $0.86-1.08$ \\
& & & & & & \\
JSRP1 & rs886363 & 0.19 & 0.20 & 0.053 & 0.91 & $0.82-1.00$ \\
JSRP1 & rs3746158 & 0.33 & 0.32 & 0.682 & 1.02 & $0.94-1.11$ \\
& & & & & & \\
UTX & rs9781530 & 0.07 & 0.06 & 0.308 & 1.09 & $0.92-1.32$ \\
UTX & rs5952647 & 0.19 & 0.18 & 0.321 & 1.06 & $0.94-1.19$ \\
UTX & rs6611063 & 0.19 & 0.19 & 0.353 & 1.03 & $0.92-1.22$ \\
UTX & rs17215160 & 0.12 & 0.12 & 0.894 & 1.07 & $0.93-1.22$ \\
UTX & rs6611065 & Failed HWE & Failed HWE & - & - & - \\
\hline
\end{tabular}

$\mathrm{Cl}$, confidence intervals; GD, Graves' disease; HWE, Hardy-Weinberg equilibrium; MAF, minor allele frequency; OR, odds ratio; SNP, single-nucleotide polymorphism. Tag SNP data for HDLBP, TEKT1, JSRP1 and UTX (phase 2, build 36, CEU population) was downloaded from the International Haplotype Mapping Project website (http://www.hapmap.org). Taking into account the initially genotyped SNPs, a further 29 Tag SNPs were chosen with a minimum $r^{2}$ of 0.80 and $\mathrm{MAF} \geqslant 0.05$ to capture the majority of common variation within these genes; including 14 for HDLBP, 8 for TEKT1 (although assays for two of these could not be designed for our genotyping platform and could not be screened), two for JSRP1 and five for UTX (see Supplementary Table 1 for SNPs captured by these 29 Tag SNPs).

All ORs are generated from the MAF. All associations are highlighted in bold.

Tag SNP genotyping results

None of the Tag SNPs for JSRP1 or UTX showed evidence of association with GD in the geographically matched combined collection of 5168 samples (2478 GD cases and 2690 controls) (Table 2). Out of 14 Tag SNPs for HDLBP; two, rs6437249 and rs11680329, showed some evidence for association with GD producing ORs of 0.91 (95\% $\mathrm{CI}=0.84-0.99)$ and $1.11(95 \% \mathrm{CI}=1.00-1.22)$, respectively. From the six Tag SNPs for TEKT1; two, rs4796561 and rs4796356, also showed some evidence for association with GD producing $\mathrm{ORs}$ of $0.88 \quad(95 \% \quad \mathrm{CI}=0.81-0.96)$ and $1.12 \quad(95 \%$ $\mathrm{CI}=1.03-1.23)$. A third SNP rs8078571 showed differences in allele frequency but no evidence of genotypic association $(P=0.104)$.

\section{DISCUSSION}

In this study, we have failed to replicate, in an adequately powered independent data set, association of nine novel nsSNPs previously reported to be weakly associated with GD in the WTCCC nsSNP study. Although none of the original nsSNPs were found to be associated with GD in the replication study, nsSNPs in HDLBP, TEKT1, JSRP1 and UTX remained weakly associated $(P<0.05)$ in the complete collection of 5924 UK GD cases and controls. Tag SNPs in HDLBP and TEKT1, not originally typed in the nsSNP study also provide a weak signal for association in the complete geographically matched collection.

A number of significant replicated associations have previously been reported for GD, the HLA class I and II regions, ${ }^{9,10}$ cytotoxic T-lymphocyte-associated protein 4 (CTLA-4), ${ }^{11}$ protein tyrosine phosphatase non-receptor type $22(P T P N 22)^{12,13}$ and $T S H R^{5}$ with ORs for the development of disease ranging from 1.50-3.00. Weaker replicated effects are also likely to be conferred by the interleukin 2 receptor alpha gene (IL2RA), ${ }^{14} C D 40^{15,16}$ and FCRL $L^{4}$ with lesser ORs of 1.10-1.30. Other loci in Caucasian subjects have been reported although findings are less consistent (thyroglobulin ${ }^{17,18}$ ) or require further replication (including PTPN2 and CD226 ${ }^{19}$ ). To help identify further susceptibility loci, large-scale genome-wide association studies (comprising >500000 SNPs) have been conducted and are at last beginning to deliver novel susceptibility loci for a number of common diseases including the autoimmune diseases. The most comprehensive association study published in GD was conducted by the WTCCC and included 14500 nsSNPs in 900 GD index cases and 1500 controls. ${ }^{4}$ Reassuringly, association with disease was replicated for the previously identified loci within the HLA region, FCRL3/5 and the TSHR. Other previously identified loci including CTLA-4 and PTPN22 were not detected as these genes were not covered in the nsSNP study, which used a custom-made Infinium array (Illumina, San Diego, CA, USA) based largely on experimentally validated nsSNPs with a MAF $>1 \%$ in western European samples. ${ }^{4}$ In addition to HLA, FCRL3/5 and the $T S H R$, nine novel regions were also reported to be weakly associated with GD at a significance level of only $P \leqslant 10^{-4}$. The lack of replication reported in the current independent collection of GD index cases and controls vindicates the caution exercised over the interpretation of the weak associations in the original WTCCC nsSNP study.

The replication collection of UK GD cases and controls used in this study was adequately powered to detect the size of effect observed in the WTCCC nsSNP study, except TEKT1. More over for the majority of SNPs tested we had $>80 \%$ power in the replication collection to detect an OR of 1.13-1.18 or higher. Clearly, however, we can not exclude smaller sized effects at these loci, which may be contributing to the overall genetic architecture of GD. Studies in other common autoimmune diseases have revealed loci conferring ORs for disease risk of $<1.20$ and have indicated the size of cohort required to detect such effects. In type 1 diabetes, wherein results from two different genome-wide studies both using approximately 4000 cases and controls found loci with small effects, including, for example, $B A C H 2$ and $\mathrm{CTSH}$, convincing genome-wide statistical significance was only achieved when these effects were tested in 12971 cases and controls. ${ }^{20}$

The replication and extension data presented in this study, based on 5924 samples and representing the largest GD association study to date, serve to highlight, the importance of appropriate levels of statistical significance and data interpretation. Our study also highlights the need for collaborative efforts to produce large collections of DNA for genome-wide screening and replication in common diseases such as GD in which individual susceptibility loci are likely to be exerting small effects.

\section{CONFLICT OF INTEREST}

The authors declare no conflict of interest. 


\section{ACKNOWLEDGEMENTS}

We would like to thank all patients, nurses and doctors for recruiting into the AITD National Collection. We wish to acknowledge the WTCCC and the Wellcome Trust for funding. We acknowledge use of DNA from the British 1958 Birth Cohort Collection, funded by the Medical Research Council grant G0000934 and the Wellcome Trust grant 068545/Z/02.

1 Tomer Y, Ban Y, Concepcion E et al: Common and unique susceptibility loci in Graves and Hashimoto diseases: results of whole-genome screening in a data set of 102 multiplex families. Am J Hum Genet 2003; 73: 736-747.

2 Taylor JC, Gough SC, Hunt PJ et al: A genome-wide screen in 1119 relative pairs with autoimmune thyroid disease. J Clin Endocrinol Metab 2006; 91: 646-653.

3 WTCCC: Genome-wide association study of 14000 cases of seven common diseases and 3000 shared controls. Nature 2007; 447: 661-678.

4 WTCCC, TACS: Association study of $14500 \mathrm{nsSNPs}$ in four common diseases identifies variants involved in autoimmunity. Nat Genet 2007; 39: 1329-1337.

5 Brand OJ, Barrett J, Simmonds MJ et al: Association of the thyroid stimulating hormone receptor gene (TSHR) with Graves' disease (GD). Hum Mol Genet 2009; 18: 1704-1713.

6 Kochi Y, Yamada R, Suzuki A et al: A functional variant in FCRL3, encoding Fc receptorlike 3 , is associated with rheumatoid arthritis and several autoimmunities. Nat Genet 2005; 37: 478-485.

7 Manji N, Carr-Smith JD, Boelaert $\mathrm{K}$ et al: Influences of age, gender, smoking, and family history on autoimmune thyroid disease phenotype. J Clin Endocrinol Metab 2006; 91: 4873-4880.

8 Mathews JD: Statistical aspects of immunogenetic association with disease. in Simons MJ, Tait BD (eds):: Detection of Immune-Associated Genetic Markers of Human Disease. London: Churchill Livingstone, 1984, pp 106-136.

9 Simmonds MJ, Howson JM, Heward JM et al: A novel and major association of HLA-C in Graves' disease that eclipses the classical HLA-DRB1 effect. Hum Mol Genet 2007; 16: 2149-2153.
10 Simmonds MJ, Howson JM, Heward JM et al: Regression mapping of association between the human leukocyte antigen region and Graves disease. Am J Hum Genet 2005; 76: 157-163.

11 Ueda $\mathrm{H}$, Howson JM, Esposito L et al: Association of the T-cell regulatory gene CTLA4 with susceptibility to autoimmune disease. Nature 2003; 423: 506-511.

12 Heward J, Brand O, Barrett J, Carr-Smith J, Franklyn J, Gough S: Association of PTPN22 haplotypes with Graves' disease. J Clin Endocrinol Metab 2006; 92 : 685-690.

13 Velaga MR, Wilson V, Jennings CE et al: The codon 620 tryptophan allele of the lymphoid tyrosine phosphatase (LYP) gene is a major determinant of Graves' disease. J Clin Endocrinol Metab 2004; 89: 5862-5865.

14 Brand OJ, Lowe CE, Heward JM et al: Association of the interleukin-2 receptor alpha $(\mathrm{IL}-2 \mathrm{R} \alpha) / \mathrm{CD} 25$ gene region with Graves' disease using a multilocus test and tag SNPs. Clin Endocrinol (Oxf) 2007; 66: 508-512.

15 Simmonds MJ, Heward JM, Franklyn JA, Gough SC: The CD40 Kozak SNP: a new susceptibility loci for Graves' disease? Clin Endocrinol (Oxf) 2005; 63: 232-233.

16 Jacobson EM, Huber AK, Akeno $\mathrm{N}$ et al: A CD40 Kozak sequence polymorphism and susceptibility to antibody-mediated autoimmune conditions: the role of CD40 tissuespecific expression. Genes Immun 2007; 8: 205-214.

17 Collins JE, Heward JM, Howson JM et al: Common allelic variants of exons 10, 12, and 33 of the thyroglobulin gene are not associated with autoimmune thyroid disease in the United Kingdom. J Clin Endocrinol Metab 2004; 89: 6336-6339.

18 Ban Y, Greenberg DA, Concepcion E, Skrabanek L, Villanueva R, Tomer Y: Amino acid substitutions in the thyroglobulin gene are associated with susceptibility to human and murine autoimmune thyroid disease. Proc Natl Acad Sci USA 2003; 100: $15119-15124$.

19 Todd JA, Walker NM, Cooper JD et al: Robust associations of four new chromosome regions from genome-wide analyses of type 1 diabetes. Nat Genet 2007; 39: $857-864$.

20 Cooper JD, Smyth DJ, Smiles AM et al: Meta-analysis of genome-wide association study data identifies additional type 1 diabetes risk loci. Nat Genet 2008; 40: 1399-1401.

\section{APPENDIX}

\section{MEMBERSHIP OF WTCCC}

Membership of the Wellcome Trust Case-Control Consortium (WTCCC)

Management Committee: Paul R Burton ${ }^{1}$, David G Clayton ${ }^{2}$, Lon R Cardon $^{3}$, Nick Craddock ${ }^{4}$, Panos Deloukas ${ }^{5}$, Audrey Duncanson ${ }^{6}$, Dominic P Kwiatkowski ${ }^{3,5}$, Mark I McCarthy ${ }^{3,7}$, Willem H Ouwehand ${ }^{8,9}$, Nilesh J Samani ${ }^{10}$, John A Todd ${ }^{2}$, Peter Donnelly (Chair) ${ }^{11}$

Analysis Committee: Jeffrey C Barrett ${ }^{3}$, Paul R Burton ${ }^{1}$, Dan Davison ${ }^{11}$, Peter Donnelly ${ }^{11}$, Doug Easton ${ }^{12}$, David Evans ${ }^{3}$, Hin-Tak Leung ${ }^{2}$, Jonathan L Marchini ${ }^{11}$, Andrew P Morris ${ }^{3}$, I CA Spencer ${ }^{11}$, Martin D Tobin ${ }^{1}$, Lon R Cardon (co-chair) ${ }^{3}$, David G Clayton (co-chair) ${ }^{2}$

UK Blood Services and University of Cambridge Controls: Antony $\mathrm{P}$ Attwood $^{5,8}$, James P Boorman ${ }^{8,9}$, Barbara Cant ${ }^{8}$, Ursula Everson ${ }^{13}$, Judith M Hussey ${ }^{14}$, Jennifer D Jolley ${ }^{8}$, Alexandra S Knight ${ }^{8}$, Kerstin Koch $^{8}$, Elizabeth Meech ${ }^{15}$, Sarah Nutland ${ }^{2}$, Christopher V Prowse ${ }^{16}$, Helen E Stevens ${ }^{2}$, Niall C Taylor ${ }^{8}$, Graham R Walters ${ }^{17}$, Neil M Walker ${ }^{2}$, Nicholas A Watkins ${ }^{8,9}$, Thilo Winzer ${ }^{8}$, John A Todd ${ }^{2}$, Willem H Ouwehand ${ }^{8,9}$

1958 Birth Cohort Controls: Richard W Jones ${ }^{18}$, Wendy L McArdle ${ }^{18}$, Susan M Ring ${ }^{18}$, David P Strachan ${ }^{19}$, Marcus Pembrey ${ }^{18,20}$

Bipolar Disorder: Aberdeen - Gerome Breen ${ }^{21}$, David St Clair ${ }^{21}$; Birmingham - Sian Caesar ${ }^{22}$, Katherine Gordon-Smith ${ }^{22,23}$, Lisa Jones ${ }^{22}$; Cardiff - Christine Fraser ${ }^{23}$, Elaine K Green ${ }^{23}$, Detelina Grozeva ${ }^{23}$, Marian L Hamshere ${ }^{23}$, Peter A Holmans ${ }^{23}$, Ian R Jones ${ }^{23}$, George Kirov ${ }^{23}$, Valentina Moskvina ${ }^{23}$, Ivan Nikolov ${ }^{23}$,

Michael C O’Donovan ${ }^{23}$, Michael J Owen ${ }^{23}$, Nick Craddock ${ }^{23}$; London - David A Collier ${ }^{24}$, Amanda Elkin ${ }^{24}$, Anne Farmer ${ }^{24}$, Richard Williamson ${ }^{24}$, Peter McGuffin ${ }^{24}$; Newcastle - Allan H Young $^{25}$, I Nicol Ferrier ${ }^{25}$

Coronary Artery Disease: Leeds - Stephen G Ball ${ }^{26}$, Anthony J Balmforth $^{26}$, Jennifer H Barrett ${ }^{26}$, D Timothy Bishop ${ }^{26}$, Mark M Iles $^{26}$, Azhar Maqbool ${ }^{26}$, Nadira Yuldasheva ${ }^{26}$, Alistair S Hall ${ }^{26}$; Leicester - Peter S Braund ${ }^{10}$, Paul R Burton ${ }^{1}$, Richard J Dixon ${ }^{10}$, Massimo Mangino ${ }^{10}$, Suzanne Stevens ${ }^{10}$, Martin D Tobin ${ }^{1}$, John R Thompson $^{1}$, Nilesh J Samani ${ }^{10}$

Crohn's Disease: Cambridge - Francesca Bredin ${ }^{27}$, Mark Tremelling ${ }^{27}$, Miles Parkes ${ }^{27}$; Edinburgh - Hazel Drummond ${ }^{28}$, Charles W Lees ${ }^{28}$, Elaine R Nimmo ${ }^{28}$, Jack Satsangi ${ }^{28}$; London - Sheila A Fisher ${ }^{29}$, Alastair Forbes ${ }^{30}$, Cathryn M Lewis ${ }^{29}$, Clive M Onnie ${ }^{29}$, Natalie J Prescott $^{29}$, Jeremy Sanderson ${ }^{31}$, Christopher G Mathew $^{29}$; Newcastle - Jamie Barbour ${ }^{32}$, M Khalid Mohiuddin ${ }^{32}$, Catherine E Todhunter ${ }^{32}$, John C Mansfield ${ }^{32}$; Oxford - Tariq Ahmad $^{33}$, Fraser R Cummings ${ }^{33}$, Derek P Jewell ${ }^{33}$

Hypertension: Aberdeen - John Webster ${ }^{34}$; Cambridge - Morris J Brown $^{35}$, David G Clayton ${ }^{2}$; Evry, France - G Mark Lathrop ${ }^{36}$; Glasgow - John Connell ${ }^{37}$, Anna Dominiczak ${ }^{37}$; Leicester - Nilesh J Samani ${ }^{10}$; London - Carolina A Braga Marcano ${ }^{38}$, Beverley Burke ${ }^{38}$, Richard Dobson ${ }^{38}$, Johannie Gungadoo ${ }^{38}$, Kate L Lee ${ }^{38}$, Patricia B Munroe $^{38}$, Stephen J Newhouse ${ }^{38}$, Abiodun Onipinla ${ }^{38}$, I Wallace ${ }^{38}$, Mingzhan Xue ${ }^{38}$, Mark Caulfield ${ }^{38}$; Oxford - Martin Farrall ${ }^{39}$

Rheumatoid Arthritis: Anne Barton ${ }^{40}$, The Biologics in RA Genetics and Genomics Study Syndicate (BRAGGS) Steering Committee, Ian $\mathrm{N}$ Bruce $^{40}$, Hannah Donovan ${ }^{40}$, Steve Eyre ${ }^{40}$, Paul D Gilbert ${ }^{40}$, Samantha L Hider ${ }^{40}$, Anne M Hinks ${ }^{40}$, Sally L John ${ }^{40}$, Catherine 
Potter $^{40}$, Alan J Silman ${ }^{40}$, Deborah PM Symmons ${ }^{40}$, Wendy Thomson $^{40}$, Jane Worthington ${ }^{40}$

Type 1 Diabetes: David G Clayton ${ }^{2}$, David B Dunger ${ }^{2,41}$, Sarah Nutland ${ }^{2}$, Helen E Stevens ${ }^{2}$, Neil M Walker², Barry Widmer²,41, John A Todd ${ }^{2}$

Type 2 Diabetes: Exeter - Timothy M Frayling ${ }^{42,43}$, Rachel M Freathy $^{42,43}$, Hana Lango ${ }^{42,43}$, John R B Perry ${ }^{42,43}$, Beverley M Shields ${ }^{43}$, Michael N Weedon ${ }^{42,43}$, Andrew T Hattersley ${ }^{42,43}$; London - Graham A Hitman $^{44}$; Newcastle - Mark Walker ${ }^{45}$; Oxford - Kate S Elliott ${ }^{3,7}$, Christopher J Groves ${ }^{7}$, Cecilia M Lindgren ${ }^{3,7}$, Nigel W Rayner ${ }^{3,7}$, Nicholas J Timpson ${ }^{3,46}$, Eleftheria Zeggini ${ }^{3,7}$, Mark I McCarthy ${ }^{3,7}$

Tuberculosis: Gambia - Melanie Newport ${ }^{47}$, Giorgio Sirugo ${ }^{47}$; Oxford - Emily Lyons ${ }^{3}$, Fredrik Vannberg ${ }^{3}$, Adrian VS Hill ${ }^{3}$

Ankylosing Spondylitis: Linda A Bradbury ${ }^{48}$, Claire Farrar ${ }^{49}$, Jennifer J Pointon ${ }^{48}$, Paul Wordsworth ${ }^{49}$, Matthew A Brown ${ }^{48,49}$

AutoImmune Thyroid Disease: Jayne A Franklyn ${ }^{50}$, Joanne M Heward $^{50}$, Matthew J Simmonds ${ }^{50}$, Stephen CL Gough ${ }^{50}$

Breast Cancer: Sheila Seal ${ }^{51}$, Breast Cancer Susceptibility Collaboration $(\mathrm{UK})^{\star}$, Michael R Stratton ${ }^{51,52}$, Nazneen Rahman ${ }^{51}$

Multiple Sclerosis: Maria $\mathrm{Ban}^{53}$, An Goris ${ }^{53}$, Stephen J Sawcer ${ }^{53}$, Alastair Compston ${ }^{53}$

Gambian Controls: Gambia - David Conway ${ }^{47}$, Muminatou Jallow $^{47}$, Melanie Newport ${ }^{47}$, Giorgio Sirugo ${ }^{47}$; Oxford - Kirk A Rockett $^{3}$, Dominic P Kwiatkowski ${ }^{3,5}$

DNA, Genotyping, Data QC and Informatics: Wellcome Trust Sanger Institute, Hinxton - Claire Bryan ${ }^{5}$, Suzannah J Bumpstead ${ }^{5}$, Amy Chaney $^{5}$, Kate Downes ${ }^{2,5}$, Jilur Ghori ${ }^{5}$, Rhian Gwilliam ${ }^{5}$, Sarah E Hunt $^{5}$, Michael Inouye ${ }^{5}$, Andrew Keniry ${ }^{5}$, Emma King ${ }^{5}$, Ralph McGinnis $^{5}$, Simon Potter ${ }^{5}$, Rathi Ravindrarajah ${ }^{5}$, Pamela Whittaker ${ }^{5}$, David Withers ${ }^{5}$, Panos Deloukas ${ }^{5}$; Cambridge - Hin-Tak Leung ${ }^{2}$, Sarah Nutland ${ }^{2}$, Helen E Stevens ${ }^{2}$, Neil M Walker ${ }^{2}$, John A Todd ${ }^{2}$

Statistics: Cambridge - Doug Easton ${ }^{12}$, David G Clayton ${ }^{2}$; Leicester Paul R Burton ${ }^{1}$, Martin D Tobin ${ }^{1}$; Oxford - Jeffrey C Barrett ${ }^{3}$, David Evans $^{3}$, Andrew P Morris ${ }^{3}$, Lon R Cardon ${ }^{3}$; Oxford - Niall J Cardin ${ }^{11}$, Dan Davison $^{11}$, Teresa Ferreira ${ }^{11}$, Joanne Pereira-Gale ${ }^{11}$, Ingeleif $\mathrm{B}$ Hallgrimsdóttir ${ }^{11}$, Bryan $\mathrm{N}$ Howie $^{11}$, Jonathan L Marchini ${ }^{11}$, I CA Spencer ${ }^{11}$, Zhan Su${ }^{11}$, Yik Ying Teo ${ }^{3,11}$, Damjan Vukcevic ${ }^{11}$, Peter Donnelly ${ }^{11}$

PIs: David Bentley ${ }^{5,54}$, Matthew A Brown ${ }^{48,49}$, Lon R Cardon ${ }^{3}$, Mark Caulfield $^{38}$, David G Clayton ${ }^{2}$, Alistair Compston ${ }^{53}$, Nick Craddock ${ }^{23}$, Panos Deloukas ${ }^{5}$, Peter Donnelly ${ }^{11}$, Martin Farrall ${ }^{39}$, Stephen CL Gough $^{50}$, Alistair S Hall ${ }^{26}$, Andrew T Hattersley ${ }^{42,43}$, Adrian VS Hill $^{3}$, Dominic P Kwiatkowski ${ }^{3,5}$, Christopher G Mathew ${ }^{29}$, Mark I McCarthy $^{3,7}$, Willem H Ouwehand ${ }^{8,9}$, Miles Parkes ${ }^{27}$, Marcus Pembrey ${ }^{18,20}$, Nazneen Rahman ${ }^{51}$, Nilesh J Samani ${ }^{10}$, Michael R Stratton $^{51,52}$, John A Todd ${ }^{2}$, Jane Worthington ${ }^{40}$

${ }^{1}$ Genetic Epidemiology Group, Department of Health Sciences, University of Leicester, Adrian Building, University Road, Leicester LE1 7RH, UK; ${ }^{2}$ Juvenile Diabetes Research Foundation/Wellcome Trust
Diabetes and Inflammation Laboratory, Department of Medical Genetics, Cambridge Institute for Medical Research, University of Cambridge, Wellcome Trust/MRC Building, Cambridge CB2 0XY, UK; ${ }^{3}$ Wellcome Trust Centre for Human Genetics, University of Oxford, Roosevelt Drive, Oxford OX3 7BN, UK; ${ }^{4}$ Department of Psychological Medicine, Henry Wellcome Building, School of Medicine, Cardiff University, Heath Park, Cardiff CF14 4XN, UK; ${ }^{5}$ The Wellcome Trust Sanger Institute, Wellcome Trust Genome Campus, Hinxton, Cambridge CB10 1SA, UK; ${ }^{6}$ The Wellcome Trust, Gibbs Building, 215 Euston Road, London NW1 2BE, UK; ${ }^{7}$ Oxford Centre for Diabetes, Endocrinology and Medicine, University of Oxford, Churchill Hospital, Oxford OX3 7LJ, UK; ${ }^{8}$ Department of Haematology, University of Cambridge, Long Road, Cambridge CB2 2PT, UK; ${ }^{9}$ National Health Service Blood and Transplant, Cambridge Centre, Long Road, Cambridge CB2 2PT, UK; ${ }^{10}$ Department of Cardiovascular Sciences, University of Leicester, Glenfield Hospital, Groby Road, Leicester LE3 9QP, UK; ${ }^{11}$ Department of Statistics, University of Oxford, 1 South Parks Road, Oxford OX1 3TG, UK; ${ }^{12}$ Cancer Research UK Genetic Epidemiology Unit, Strangeways Research Laboratory, Worts Causeway, Cambridge CB1 8RN, UK; ${ }^{13}$ National Health Service Blood and Transplant, Sheffield Centre, Longley Lane, Sheffield S5 7JN, UK; ${ }^{14}$ National Health Service Blood and Transplant, Brentwood Centre, Crescent Drive, Brentwood CM15 8DP, UK; ${ }^{15}$ The Welsh Blood Service, Ely Valley Road, Talbot Green, Pontyclun CF72 9WB, UK; ${ }^{16}$ The Scottish National Blood Transfusion Service, Ellen's Glen Road, Edinburgh EH17 7QT, UK; ${ }^{17}$ National Health Service Blood and Transplant, Southampton Centre, Coxford Road, Southampton SO16 5AF, UK; ${ }^{18}$ Avon Longitudinal Study of Parents and Children, University of Bristol, 24 Tyndall Avenue, Bristol BS8 1TQ, UK; ${ }^{19}$ Division of Community Health Services, St George's University of London, Cranmer Terrace, London SW17 0RE, UK; ${ }^{20}$ Institute of Child Health, University College London, 30 Guilford St, London WC1N 1EH, UK; ${ }^{21}$ University of Aberdeen, Institute of Medical Sciences, Foresterhill, Aberdeen AB25 2ZD, UK; ${ }^{22}$ Division of Neuroscience, Department of Psychiatry, Birmingham University, Birmingham B15 2QZ, UK; ${ }^{23}$ Department of Psychological Medicine, Henry Wellcome Building, School of Medicine, Cardiff University, Heath Park, Cardiff CF14 4XN, UK; ${ }^{24}$ King's College London, SGDP, The Institute of Psychiatry, De Crespigny Park Denmark Hill London SE5 8AF, UK; ${ }^{25}$ School of Neurology, Neurobiology and Psychiatry, Royal Victoria Infirmary, Queen Victoria Road, Newcastle upon Tyne NE1 4LP, UK; ${ }^{26}$ Faculty of Medicine and Health, LIGHT and LIMM Research Institutes, University of Leeds, Leeds LS1 3EX, UK; ${ }^{27} \mathrm{IBD}$ Research Group, Addenbrooke's Hospital, University of Cambridge, Cambridge CB2 2QQ, UK; ${ }^{28}$ Gastrointestinal Unit, School of Molecular and Clinical Medicine, University of Edinburgh, Western General Hospital, Edinburgh EH4 2XU, UK; ${ }^{29}$ King's College London School of Medicine, Department of Medical and Molecular Genetics, 8th Floor Guy's Tower, Guy's Hospital, London SE1 9RT, UK; ${ }^{30}$ Institute for Digestive Diseases, University College London Hospitals Trust, London NW1 2BU, UK; ${ }^{31}$ Department of Gastroenterology, Guy's and St Thomas' NHS Foundation Trust, London SE1 7EH, UK; ${ }^{32}$ Department of Gastroenterology and Hepatology, University of Newcastle upon Tyne, Royal Victoria Infirmary, Newcastle upon Tyne NE1 4LP, UK; ${ }^{33}$ Gastroenterology Unit, Radcliffe Infirmary, University of Oxford, Oxford OX2 6HE, UK; ${ }^{34}$ Medicine and Therapeutics, Aberdeen Royal Infirmary, Foresterhill, Aberdeen, Grampian AB9 2ZB, UK; ${ }^{35}$ Clinical Pharmacology Unit and the Diabetes and Inflammation Laboratory, University of Cambridge, Addenbrookes Hospital, Hills Road, Cambridge CB2 2QQ, UK; ${ }^{36}$ Centre National de Genotypage, 2, Rue Gaston Cremieux, 
Evry, Paris 91057; ${ }^{37}$ BHF Glasgow Cardiovascular Research Centre, University of Glasgow, 126 University Place, Glasgow G12 8TA, UK; ${ }^{38}$ Clinical Pharmacology and Barts and The London Genome Centre, William Harvey Research Institute, Barts and The London, Queen Mary's School of Medicine, Charterhouse Square, London EC1M 6BQ, UK; ${ }^{39}$ Cardiovascular Medicine, University of Oxford, Wellcome Trust Centre for Human Genetics, Roosevelt Drive, Oxford OX3 7BN, UK; ${ }^{40}$ arc Epidemiology Research Unit, University of Manchester, Stopford Building, Oxford Rd, Manchester M13 9PT, UK; ${ }^{41}$ Department of Paediatrics, University of Cambridge, Addenbrooke's Hospital, Cambridge CB2 2QQ, UK; ${ }^{42}$ Genetics of Complex Traits, Institute of Biomedical and Clinical Science, Peninsula Medical School, Magdalen Road, Exeter EX1 2LU UK; ${ }^{43}$ Diabetes Genetics, Institute of Biomedical and Clinical Science, Peninsula Medical School, Barrack Road, Exeter EX2 5DU UK; ${ }^{44}$ Centre for Diabetes and Metabolic Medicine, Barts and The London, Royal London Hospital, Whitechapel, London E1 1BB UK; ${ }^{45}$ Diabetes Research Group, School of Clinical Medical Sciences,
Newcastle University, Framlington Place, Newcastle upon Tyne NE2 $4 \mathrm{HH}, \mathrm{UK} ;{ }^{46}$ The MRC Centre for Causal Analyses in Translational Epidemiology, Bristol University, Canynge Hall, Whiteladies Rd, Bristol BS2 8PR, UK; ${ }^{47}$ MRC Laboratories, Fajara, The Gambia; ${ }^{48}$ Diamantina Institute for Cancer, Immunology and Metabolic Medicine, Princess Alexandra Hospital, University of Queensland, Woolloongabba, Qld 4102, Australia; ${ }^{49}$ Botnar Research Centre, University of Oxford, Headington, Oxford OX3 7BN, UK; ${ }^{50}$ Division of Medical Sciences, Department of Medicine, Institute of Biomedical Research, University of Birmingham, Edgbaston, Birmingham B15 2TT, UK; ${ }^{51}$ Section of Cancer Genetics, Institute of Cancer Research, 15 Cotswold Road, Sutton SM2 5 NG, UK; ${ }^{52}$ Cancer Genome Project, The Wellcome Trust Sanger Institute, Wellcome Trust Genome Campus, Hinxton, Cambridge CB10 1SA, UK; ${ }^{53}$ Department of Clinical Neurosciences, University of Cambridge, Addenbrooke's Hospital, Hills Road, Cambridge CB2 2QQ, UK; ${ }^{54}$ PRESENT ADDRESS: Illumina Cambridge, Chesterford Research Park, Little Chesterford, Nr Saffron Walden, Essex CB10 1XL, UK.

Supplementary Information accompanies the paper on European Journal of Human Genetics website (http://www.nature.com/ejhg) 